\title{
Dependencia alcohólica con y sin trastornos psiquiátricos asociados
}

\author{
Cuadrado Callejo, P. \\ Psiquiatra. Coordinador del Programa de Problemas Relacionados con el Alcohol. \\ Servicio de Salud Mental Retiro. Consejería de Sanidad. Comunidad de Madrid.
}

Enviar correspondencia a:

Pedro Cuadrado Callejo. SSM Retiro. C/ Doctor Castelo 60. 28009 Madrid. Tfno. 915868524. Fax 915868763. Correo electrónico: Pedrocuadrado@Reteline.es

\section{RESUMEN}

Objetivos. Determinar si la presencia de comorbilidad influye en la respuesta al tratamiento y evolución asi como si es un criterio útil para establecer subtipos dentro de la dependencia alcohólica. Diseño. Se compararon dos submuestras de dependientes del alcohol: $A(n=120)$ con comorbilidad, $B(n=41) \sin$ comorbilidad, en función de variables sociodemográficas, clínicas, respuesta al tratamiento y evolución 5-7 años. Setting. Programa de tratamiento ambulatorio de problemas relacionados con el alcohol en un servicio de salud mental de Madrid. Participantes. 161 casos nuevos que iniciaron tratamiento entre 1989 y 1991, con un diagnóstico de dependencia alcohólica. Medidas. Criterios diagnósticos DSM III-R, patrones de consumo según Miller y Marlatt, cuestionario de personalidad EPO (A), tasas de abandono y tasas de abstinencia absoluta como criterios de respuesta al tratamiento y evolución. Hallazgos. Una menor edad en los dependientes del alcohol con comorbilidad. No diferencias en las características clínicas, respuesta al tratamiento y evolución entre dependientes con y sin comorbilidad asociada. Conclusiones. La comorbilidad en su conjunto no influye en la respuesta al tratamiento y evolución ni es un criterio útil para establecer subtipos dentro de la dependencia alcohólica.

Palabras clave: DEPENDENCIA ALCOHOLICA. COMORBILIDAD. PRONOSTICO. RESULTADOS.

\section{INTRODUCCION}

B astantes trabajos se han centrado en la asociación de la dependencia alcohólica a algunos trastornos psiquiátricos, sobre todo la depresión, desde la distinción de lo primario y secundario $(1,2)$, y más recientemente desde la distinción entre los trastornos afectivos independientes y los inducidos por el alcohol (3), la personalidad antisocial $(4,5)$, los trastornos de ansiedad (6), o los trastornos de la alimentación (7). Sin embargo, tanto los estudios comunitarios

\section{SUMMARY}

Aim. Determine if the presence of co-morbidity influences long term outcome and evolution and if this criterion is useful to establish subtypes within alcohol dependence. Design: Two subsamples of alcohol dependent subjects were compared: alcohol: $A(n=120)$ with co-morbidity, $B(n=41)$ without comorbidity, based on sociodemographic, clinical characteristics, treatment response and 5-7 year evolution variables. Setting. Outpatient treatment program of alcohol related problems in a mental health service of Madrid. Participants. 161 new cases diagnosed of alcohol dependence who initiated treatment between 1989 and 1991. Measurements. DSM-III-R diagnostic criteria, consumption standards according to Miller and Marlatt, EPQ personality questionnaire (A), drop-out and absolute abstinence rates as outcome criteria. Findings. Age is lower in alcohol dependent subjects with co-morbidity and no differences are found in clinical characteristics, response to treatment and evolution between dependent subjects with and without associated co-morbidity. Conclusions. Co-morbidity as a whole did not influence in long term outcome and it is not a useful criterion to establish subtypes within alcohol dependence.

Key words: ALCOHOL DEPENDENCE. COMORBIDITY. PROGNOSIS.TREATMENT OUTCOME.

$(8,9,10,11)$, como los clínicos $(12,13,14,15)$, ponen de manifiesto que la comorbilidad en la dependencia alcohólica se da con todos los trastornos psiquiátrico, aunque con unos con más frecuencia que con otros.

En el estudio de las implicaciones de la comorbilidad en su conjunto sobre la clínica y evolución de la dependencia alcohólica, son pioneros los trabajos del grupo de Kansas en alcohólicos ingresados (16), sobre todo en la idea de utilizar la comorbilidad en su conjunto como criterio para clasificar a los alcohólicos, estableciendo dos tipos: alcoholismo primario o no 
complicado y alcoholismo combinado, que más adelante van a redefinir como alcoholismo monosindrómico y multisindrómico $(17,18,19)$. Sus estudios sobre las características clínicas y evolución a corto plazo (17), y estabilidad de los trastornos asociados (19), les lleva a considerar como demostrado que el comienzo, severidad, resultados e historia familiar de los alcohólicos monosindrómicos difiere significativamente de la de los alcohólicos multisindrómicos $(18,19)$. Sin embargo, los resultados de distintos trabajos acerca de las características sociodemográficas, clínicas y de respuesta al tratamiento y evolución ofrecen un panorama diferente, sobre todo cuando se centran en un trastorno psiquiátrico determinado.

\section{Características sociodemográficas.}

Se ha descrito más comorbilidad en las mujeres $(9$, $20,21)$, algunos grupos de edad (9), solteros (22), y menor nivel socioeconómico y educacional $(22,9)$. Sin embargo, otros trabajos no encuentran diferencias con respecto a estas características $(23,18)$. Kessler y otros (9), analizando los datos del National Comorbidity Survey, encuentran mayores tasas de comorbilidad para todos los trastornos entre 25 y 34 años. Sin embargo, cuando se analiza esta relación en función del tipo de trastorno asociado, se ha descrito que el ser más joven se da en la asociación a trastornos por drogas, trastornos de la personalidad y esquizofrenia (2, $22,24)$, mientras que la asociación a trastornos afectivos se da en los dependientes de más edad (24). En relación al sexo, Tomasson y Vaglum (14), no encuentran diferencias para la comorbilidad en su conjunto, y en el análisis por trastornos, señalan, como otros autores $(14,25,26)$, que, en los dependientes del alcohol, la distribución de los tipos de trastornos asociados es igual a la que se da en la población general.

\section{Características clínicas.}

Aquellos con comorbilidad tendrían un comienzo más precoz de la dependencia alcohólica $(12,18,27$, 28), más problemas relacionados (18),y más severidad medida con el MAST $(12,22)$, más historia familiar de alcoholismo $(18,27)$, y más historia familiar de otros trastornos psiquiátricos (18).

El análisis por trastornos pone de manifiesto que algunos hallazgos, atribuidos a la comorbilidad en su conjunto, son específicos de algunos trastornos pero no de otros. Así, el comienzo más temprano de la dependencia aparece ligado a la asociación a personalidad antisocial $(4,12,5,29,30)$,y trastornos por drogas $(12,21)$, y a algún trastorno afectivos (31);pero no a todos en su conjunto $(1,12)$. En el caso de la grave- dad de la dependencia ocurre lo mismo: más gravedad para la asociación a trastornos por drogas y personalidad antisocial $(32,4,12,33,5,29)$ pero no diferencias para la asociación a trastornos afectivos (12),y algún tipo de trastorno de personalidad $(12,34)$.

En el tema de la agregación familiar, es muy esclarecedor el trabajo de Kendler y otros (35), que analizando los datos del National Comorbidity Survey, encuentran que si se controla la presencia de los otros trastornos psiquiátricos en el familiar estudiado, la presencia de dependencia alcohólica, con ó sin comorbilidad, no se asocia a más agregación familiar con ningún trastorno psiquiátrico excepto con la dependencia alcohólica.

\section{Respuesta al tratamiento y evolución.}

Hay un gran acuerdo en la mayoría de los autores acerca de la existencia de más historia de tratamientos previos en los dependientes del alcohol con comorbilidad $(1,12,36,23,18,27,37)$, y de más necesidades asistenciales (38).

En cuanto a la respuesta al tratamiento y evolución, hay datos de seguimientos cortos, donde los resultados varían en función del criterio de evaluación utilizado. Se ha descrito una peor evolución, con un peor funcionamiento general en aquellos con comorbilidad asociada (18), y en varones pero no en mujeres (39). Sin embargo, utilizando las tasas de abstinencia como criterio de evaluación, los resultados son bastante unánimes, en el sentido de que la comorbilidad en su conjunto no modifica la evolución de la dependencia alcohólica a corto plazo (17, 12, 39, 27).

Al igual que en las características sociodemográficas y clínicas, el tipo de trastorno asociado va a influir en los resultados. Así, se ha encontrado peor evolución para la asociación a determinados trastornos de la personalidad $(40,41)$, y a determinados trastornos por uso de sustancias (42), pero no para la depresión $(35,39,43,44,45)$.

El objetivo del presente estudio es replicar los trabajos del grupo de Kansas, en una muestra española de dependientes del alcohol en tratamiento ambulatorio, y comprobar si los resultados obtenidos apoyan la hipótesis de la validez de la comorbilidad como criterio para subtipificar pacientes con dependencia alcohólica.

\section{MUESTRA Y METODOS}

\section{Muestra.}

La muestra procede de los 181 casos nuevos remitidos al programa de Problemas Relacionados con el 
Alcohol del Servicio de Salud Mental Retiro, Madrid, durante los años 1989, 1990 y 1991. En 20 casos no se pudo recoger los datos del protocolo, quedando la muestra reducida a 161 pacientes con dependencia alcohólica con criterios DSM III-R.

\section{Evaluación al entrar en tratamiento.}

Los pacientes son evaluados al entrar en tratamiento mediante una entrevista de recepción que llevan a cabo un trabajador social y un ATS que permite la recogida de datos sociodemográficos y donde se instruye al paciente para la realización del cuestionario de personalidad de Eysenck para adultos (EPQ-A) (46), y varias entrevistas clínicas que lleva a cabo el psiquiatra responsable del tratamiento y seguimiento del paciente, donde se recogen datos referentes a la historia de consumo, patrón de consumo y complicaciones del mismo, así como la presencia de síntomas de dependencia alcohólica y severidad de la misma, y presencia de síntomas de trastornos psiquiátricos asociados.

Para clasificar los patrones de consumo se utilizaron los criterios de Miller \& Marlatt (47), modificados. Se consideró como patrón "estable", al consumo diario por encima de 4 unidades/día y no presencia de embriagueces. En el patrón "intermitente" se alternan períodos de abstinencia o consumo inferior a 4 unidades/día con uno o más días de aumento del consumo de alcohol que se acompaña de embriaguez. El patrón "combinado" se caracteriza por consumo diario por encima de 4 unidades, junto a la presencia de embriagueces.

Para el diagnóstico de la dependencia alcohólica y los trastornos mentales asociados, se utilizaron los criterios diagnósticos propuestos por la DSM III-R, sin aplicación de criterios jerárquicos de exclusión, y utilizando, para los trastornos asociados, toda la vida del sujeto como período de evaluación (comorbilidad a lo largo de la vida). Se ha establecido el comienzo de la dependencia cuando estaban presentes más de tres criterios DSM III R, utilizando, también,los criterios DSM III-R para establecer la gravedad de la dependencia alcohólica. Se ha considerado historia familiar de dependencia alcohólica, cuando se referían síntomas de esta enfermedad en al menos un familiar de primer grado.

\section{Tratamiento recibido y respuesta al tratamiento.}

Todos los pacientes se incorporan a un programa de tratamiento ambulatorio de dos años de duración, como mínimo, que cubre las distintas fases del proceso de tratamiento, con un amplio espectro de técnicas utilizadas, cuyos componentes son: terapia individual, tratamiento farmacológico con interdictores y farma- coterapia específica para cada trastorno asociado, programa de prevención de recaídas, intervención familiar y coordinación con grupos de autoayuda, atención primaria de salud y unidades de hospitalización, cuyo desarrollo ha sido descrito más ampliamente en otro lugar (48).

Se cuantificó el tratamiento a través del número de consultas recibidas por paciente, meses de contacto con el programa, inclusión en el programa de prevención de recaídas y tratamiento en régimen de internamiento. Se establecieron cinco categorías para el tratamiento farmacológico, tanto para el tratamiento con aversivos como para antidepresivos: no uso, uso menos de seis meses, uso entre seis meses y un año, uso entre uno y dos años, uso más de dos años.

Con los datos recogidos en la historia clínica se llevó a cabo la evaluación de la respuesta al tratamiento a los seis meses, y al año, en aquellos pacientes que seguían en contacto con el programa en esos momentos. Se utilizó como criterio de resultados la abstinencia absoluta de alcohol en los últimos tres meses para la evaluación a los seis meses y en los últimos seis meses para la evaluación al año.

Para la evaluación de la respuesta al tratamiento a los dos años se utilizaron dos criterios: la permanencia en el programa, donde se establecieron cinco categorías (no abandono, abandono antes del mes, abandono entre uno y seis meses, abandono entre seis meses y un año y abandono entre uno y dos años), y la abstinencia absoluta de alcohol en el año previo a la evaluación.

\section{Evaluación tras 5 a 7 años.}

Entre el 20 de noviembre y el 31 de diciembre de 1996, tras 5 a 7 años desde el inicio del tratamiento, con una media de 70 meses, el mismo psiquiatra que había realizado el tratamiento

llevó a cabo una nueva evaluación de la muestra. No se pudo contactar con 36 casos, obteniéndose datos de 125 pacientes. Para la evaluación de la evolución a los 5-7 años se utilizó como criterio la abstinencia absoluta en el último año.

\section{Análisis estadístico.}

Se llevó a cabo un análisis bivariado para estudiar la asociación de las características socio-demográficas, clínicas, tratamiento recibido, respuesta al tratamiento y evolución 5-7 años con la presencia de comorbilidad en la dependencia alcohólica, utilizándose Chi cuadrado, con corrección Yates para aquellos análisis con número de casos inferior a 5, para las variables nominales, y t de student para las variables continuas. En un segundo momento, se realizó un análisis multivariado de regresión logística en el que se incluyeron 
como variables independientes aquellas cuya p en el análisis bivariado era inferior a 0.05 y como variable resultado la presencia de comorbilidad en la dependencia alcohólica, considerándose una asociación significativa para el 95\%, cuando la unidad estuviese incluida en el intervalo de confianza. Todos los análisis estadísticos fueron realizados con el paquete informático SPSS.PC versión 3.1 (49).

\section{RESULTADOS}

\section{Características de la muestra.}

En la muestra, un $84 \%$ eran varones, con una edad media de 40.8 años. El $57 \%$ estaban casados y el $18.6 \%$ separados. Un $39 \%$ de la muestra se situaba en el nivel socio-económico medio bajo, un $24 \%$ en el nivel medio medio, un 19\% en el nivel bajo y un $18 \%$ en el nivel medio alto. Con respecto a la situación laboral,un $67 \%$ estaban trabajando frente a un $22 \%$ que estaban sin empleo. En cuanto al nivel educativo, un $37 \%$ había finalizado estudios primarios, un $25 \%$ secundarios, un $17 \%$ estudios superiores y un $21 \%$ no poseían ningún estudio.

En cuanto a las características de la dependencia, la edad media de inicio del consumo era a los 16.5 años, con un rango entre 7 y 39 años. En el 43.4\%, el inicio de la dependencia alcohólica fue anterior a los 25 años y la duración media de la misma era de 13.1 años. Un $81 \%$ de los pacientes presentaban una severidad de la dependencia "grave", un 74.5\% tuvo un patrón de consumo "combinado", un 13.6\% un patrón "estable" y un $11.8 \%$ un patrón "intermitente". Hubo complicaciones somáticas en el $81.4 \%$, complicaciones sociales en el $96.9 \%$ y problemas legales en el $43.5 \%$. En relación con la comorbilidd, se diagnosticaron 208 trastornos psiquiátricos asociados a lo largo de la vida que se agruparon en 120 pacientes, cuyos resultados se recogen en la TABLA 1. El 75\% de los pacientes presentaron al menos un trastorno psiquiátrico asociado a lo

\section{TABLA 1.- COMORBILIDAD PSIQUIÁTRICA A LO LARGO DE LA VIDA}

\begin{tabular}{lrr}
\hline NTOTAL = 161 & N & \% \\
\hline ALGÚN TRASTORNO PSIOUIÁTRICO & 120 & $75 \%$ \\
TRASTORNOS AFECTIVOS & 72 & $45 \%$ \\
TRASTORNOS DE PERSONALIDAD & 60 & $37 \%$ \\
TRASTORNOS POR DROGAS & 26 & $16 \%$ \\
JUEGO PATOLÓGICO & 12 & $7 \%$ \\
TRASTORNOS DE ANSIEDAD & 12 & $7 \%$ \\
TRASTORNOS PARANOIDES & 11 & $7 \%$ \\
ESOUIZOFRENIA & 3 & $2 \%$ \\
DEMENCIAS & 3 & $2 \%$ \\
TRASTORNOS DE ALIMENTACIÓN & 1 & $0^{\prime} 6 \%$ \\
& &
\end{tabular}

largo de su vida, siendo los trastornos afectivos, los trastornos de personalidad y los trastornos por otras drogas, los que se asocian con más frecuencia.

\section{Respuesta al tratamiento y evolución.}

A los seis meses, el análisis se llevó a cabo sobre 124 pacientes que permanecían en tratamiento, que se compararon con los 37 que habían abandonado el mismo no encontrándose diferencias en las variables sociodemográficas y clínicas. La respuesta, al año de tratamiento, se estudió en 102 pacientes que seguían en el programa, que se compararon con los 59 que lo habían abandonado,encontrándose menor duración de la dependencia y más severidad en los que abandonaron. A los dos años, se hizo el análisis sobre 133 pacientes, 72 seguían en el programa y a los otros 61 se les hizo una entrevista telefónica, no existiendo diferencias entre ambas submuestras en cuanto a la respuesta al tratamiento, y tampoco cuando se compararon con los 28 pacientes con los que no se pudo contactar.

En la evolución 5-7 años, de la muestra original, 13 habían fallecido, 39 seguían en contacto con el programa y se les hizo una entrevista cara a cara, y a 73 a través de una entrevista telefónica, no existiendo diferencias significativas en la evolución de las dos submuestras (50), y en 36 casos no se pudo realizar el contacto, por lo que el análisis se llevo a cabo sobre 112 pacientes. Para comprobar la representatividad de esta submuestra, se comparó con la submuestra de 49 pacientes de los que no se tenía de datos. En este último grupo había una mayor presencia de varones, nivel de instrucción secundario y más complicaciones somáticas; no existiendo diferencias en el resto de las variables sociodemográficas y clínicas.

\section{Dependencia alcohólica con comorbilidad versus Dependencia alcohólica sin comorbilidad.}

120 dependientes del alcohol cumplían criterios para otro trastorno psiquiátrico a lo largo de su vida, constituyendo el grupo A: dependencia alcohólica con comorbilidad, y 41 no cumplían criterios, constituyendo el grupo B: dependencia alcohólica sin comorbilidad.

En el análisis bivariado, de las variables sociodemográficas, existen diferencias significativas en la edad y en el nivel de instrucción (Tabla 2). Se encuentra que los dependientes del alcohol con comorbilidad, son más jóvenes y con un mayor nivel de instrucción; mientras que el ser mujer aumenta mucho las probabilidades de tener comorbilidad pero no llega a la significación estadística. De las características clínicas, los dependientes con comorbilidad presentan una menor duración de la dependencia y una mayor presencia de patrón de consumo intermitente, así como menores puntuaciones en extroversión (Tabla 3). En 
TABLA 2.- VARIABLES SOCIODEMOGRAFICAS

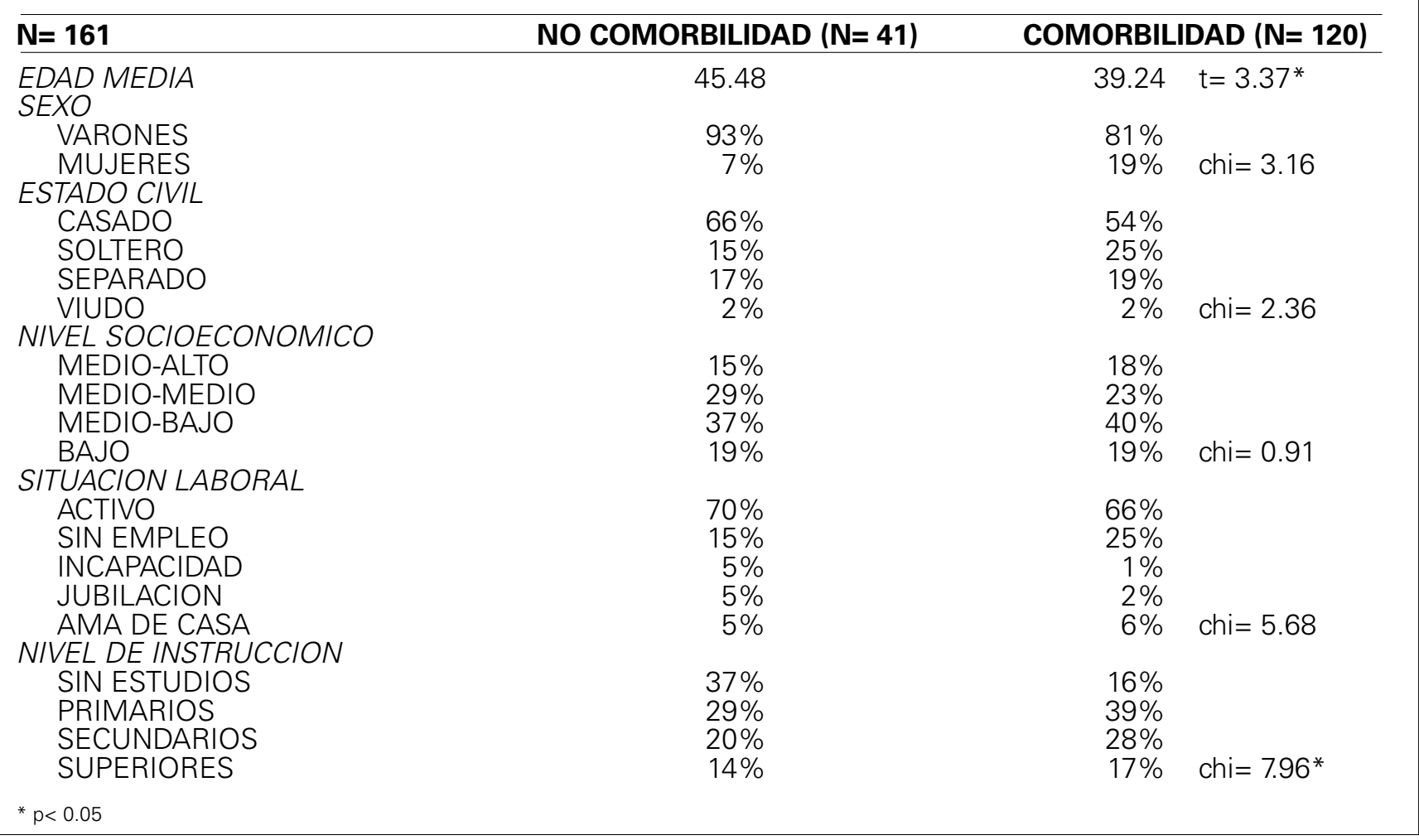

\section{TABLA 3.- VARIABLES CLÍNICAS}

\section{$\mathbf{N}=161$}

INICIO DEL CONSUMO INICIO DE DEPENDENCIA ANTES 25 AÑOS

DESPUES 25 ANNOS

DURACION DE DEPENDENCIA

PATRON DE CONSUMO

ESTABLE

INTERMITENTE

COMBINADO

SEVERIDAD

LIGERA

MODERADA

GRAVE

PROBLEMAS SOMATICOS

PRESENCIA

NO PRESENCIA

PROBLEMAS SOCIALES

PRESENCIA

NO PRESENCIA

PROBLEMAS LEGALES

PRESENCIA

NO PRESENCIA

$H^{a}$ FAMILIAR DEPENDENCIA

PRESENCIA

NO PRESENCIA

$H^{a}$ FAMILIAR T.MENTALES

PRESENCIA

NO PRESENCIA

PERSONALIDAD (EPQ-A)

NEUROTICISMO

EXTROVERSION

PSICOTICISMO
NO COMORBILIDAD ( $\mathrm{N}=\mathbf{4 1}$ )

16.48

$34 \%$

$66 \%$

17.24

$27 \%$

$0 \%$

$73 \%$

$5 \%$

$12 \%$

$83 \%$

$90 \%$

$10 \%$

$95 \%$

$5 \%$

$39 \%$

$61 \%$

$46 \%$

$54 \%$

$17 \%$

$83 \%$

15.43

13.17

4.12
COMORBILIDAD ( $\mathrm{N}=120)$

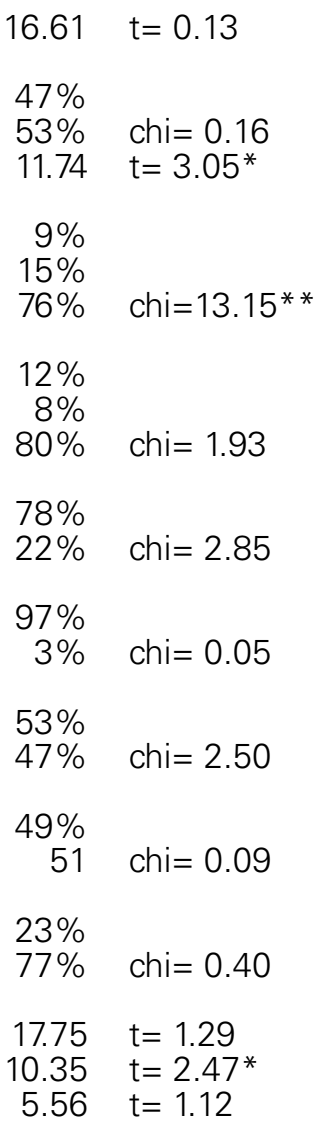

${ }^{*} p<0.05 ;{ }^{*} p<0.01$ 
cuanto al tratamiento recibido, en aquellos con comorbilidad se utilizan más antidepresivos y tienen más historia de tratamientos previos para la dependencia, también requieren mayor número de consultas y se mantienen más tiempo en contacto con el programa, pero no llega a la significación estadística (TABLA 4).

En la respuesta al tratamiento (TABLA 5), no se encontraron diferencias significativas en cuanto a las tasas de abandono entre aquellos con y sin comorbilidad. En la respuesta al tratamiento, a los seis meses, se encontraron menores tasas de abstinencia en aquellos con comorbilidad asociada; al año, no se encontraron diferencias significativas entre ambos grupos, y lo mismo ocurrió en la respuesta al tratamiento a los dos años. En la evolución 5-7 años, tampoco se encontraron diferencias significativas, en las tasas de abstinencia en el último año, entre aquellos con y sin comorbilidad (TABLA 5).

Para el análisis multivariado, se llevó a cabo una regresión logística, utilizando como variables independientes aquellas cuya $\mathrm{p}$ en el análisis bivariado era inferior a 0.05: edad, nivel de instrucción, duración de la dependencia, patrón de consumo, extroversión, uso de antidepresivos e historia de tratamientos previos, y como variable resultado la presencia de comorbilidad asociada. Se encuentra que una vez que se controla la edad, el resto de las variables no se asocia significativamente a la presencia de comorbilidad.

\section{DISCUSION}

\section{Características sociodemográficas y clínicas.}

En el análisis bivariado, únicamente la edad y el nivel de instrucción aparecen significativamente asociados a la presencia de un trastorno psiquiátrico asociado. Sin embargo,la asociación de un mayor nivel de instrucción a la presencia de comorbilidad parece un efecto de la menor edad del grupo con comorbilidad, y el nivel de instrucción, cuando se controla esta variable en la regresión logística, pierde su significación estadística. La mayor presencia de comorbilidad, en los dependientes del alcohol más jóvenes, es un dato refrendado en la literatura. Así, Kessler y otros (9), analizando los datos del National Comorbidity Survey, encuentran mayores tasas de comorbilidad para todos los trastornos entre 25 y 34 años. Además, esta asociación es más específica para determinados trastornos psiquiátricos como los trastornos por drogas, trastornos de la personalidad y esquizofrenia $(2,22$, 24), y en nuestro estudio, la asociación a este grupo de trastornos, afecta a más de la mitad de la muestra. Aunque hay una mayor presencia de comorbilidad en el sexo femenino que en el masculino, ésta no llega a la significación estadística, en línea con los resultados de Tomasson y Vaglum (14). Se puede concluir, que la presencia de comorbilidad en su conjunto no introduce características sociodemográficas específicas.

En cuanto a las características clínicas, encontramos que la presencia de comorbilidad se asocia a una menor duración de la dependencia, al patrón de consumo intermitente y a mayores puntuaciones en extroversión en el EPQ (A), en el análisis bivariado. Sin embargo, en el análisis multivariado, cuando se controla la edad, estas variables no tienen una asociación estadísticamente significativa con la presencia de comorbilidad. Estos resultados van en línea con lo publicado por otros autores $(1,12)$, que, estudiando la comorbilidad en su conjunto, no encuentran que las características clínicas permitan diferenciar a los dependientes del alcohol con y sin comorbililidad. Frente a los hallazgos del grupo de Kansas $(18,27)$, en nuestra muestra, la comorbilidad no se asocia a más historia familiar de alcoholismo, apoyando los hallazgos de Kendler y otros (35) en su análisis de los datos del National Comorbidity Survey. Por lo tanto, en nuestro trabajo, no se encuentran características clínicas específicas para la dependencia alcohólica con comorbilidad.

\section{Respuesta al tratamiento y evolución.}

En el grupo con comorbilidad, encontramos más historia de tratamientos previos para la dependencia alcohólica, dato muy refrendado en la literatura $(1,12$, $36,23,18,27,37)$. Aquellos con comorbilidad presentan más meses de contacto con el programa y mayor número de consultas, pero no llega a la significación estadística. Aunque las características de los programas, su intensidad y la duración de los mismos, puede condicionar los resultados, parece lógico que la presencia de comorbilidad va a implicar mayores necesidades asistenciales, como han puesto de relieve otros autores (38). El mayor uso de antidepresivos es un resultado obvio, dado que su uso estaba ligado a la presencia de trastornos afectivos, trastornos por angustia y trastornos por control de impulsos.

En cuanto a la respuesta al tratamiento y evolución, en nuestra muestra, la presencia de un trastorno asociado no influyó en la permanencia y cumplimiento del tratamiento, y utilizando las tasas de abstinencia como criterio de evaluación, únicamente a los seis meses hay una peor respuesta al tratamiento en el grupo con comorbilidad, que desaparece al año y a los dos años, en los que no se encuentran diferencias entre ambos grupos, y lo mismo ocurre en la evolución 5-7 años. Datos similares encuentran otros autores, cuando utilizan las tasas de abstinencia como criterio de evaluación $(17,12,39,27)$. La existencia de estudios con resultados opuestos a los señalados, sobre todo para distintos trastornos de la personalidad $(40,41)$ y algunos trastornos por uso de sustancias 
TABLA 4.- TRATAMIENTO RECIBIDO

\section{$\mathrm{N}=161$}

NUMERO DE CONSULTAS

MESES DE CONTACTO

USO DE INTERDICTORES

NO USO

MENOS DE 6 MESES

ENTRE 6 MESESY 1 AÑO

ENTRE 1 Y 2 AÑOS

MAS DE 2 AÑOS

USO DE ANTIDEPRESIVOS

NO USO

MENOS DE 6 MESES

ENTRE 6 MESESY 1 AÑO

ENTRE 1 Y 2 AÑOS

MAS DE 2 AÑOS

PREVENCION DE RECAIDAS

$\mathrm{SI}$

NO

UNIDAD DE DESHABITUACION

$\mathrm{SI}$

NO

GRUPOS DE AUTOYUDA

$\mathrm{SI}$

NO

TRATAMIENTOS PREVIOS

$\mathrm{SI}$

NO
NO COMORBILIDAD ( $\mathrm{N}=41)$

15.82

24.09

$27 \%$

$29 \%$

$22 \%$

$17 \%$

$5 \%$

$91 \%$

$7 \%$

$0 \%$

$0 \%$

$2 \%$

$32 \%$

$68 \%$

$5 \%$

$95 \%$

$20 \%$

$80 \%$

$37 \%$

$63 \%$
COMORBILIDAD ( $\mathrm{N}=120)$

$20.93 t=1.55$

$30.70 t=1.42$

$36 \%$

$28 \%$

$10 \%$

$12 \%$

$14 \% \quad$ chi $=7.01$

$67 \%$

$12 \%$

$7 \%$

$4 \%$

$10 \%$ chi $=9.67 *$

$28 \%$

$72 \% \quad$ chi $=0.16$

$16 \%$

$84 \% \quad$ chi $=3.23$

$18 \%$

$82 \% \quad$ chi $=0.08$

$62 \%$

$38 \%$ chi $=7.77^{*} *$

** $p<0.01$

TABLA 5.- RESPUESTA AL TRATAMIENTO Y EVOLUCIÓN

\section{NO COMORBILIDAD}

ABANDONO DEL TRATAMIENTO NO ABANDONO

ABANDONO ANTES DE 1 MES

ABANDONO ENTRE 1 Y 6 MESES

ENTRE 6 MESESY 1 AÑO

ENTRE 1 Y 2 AÑOS

RESPUESTA 6 MESES

ABSTINENCIA

NO ABSTINENCIA

RESPUESTA 1 AÑO

ABSTINENCIA

NO ABSTINENCIA

RESPUESTA 2 AÑOS

ABSTINENCIA

NO ABSTINENCIA

EVOLUCION 5-7 AÑOS

ABSTINENCIA

NO ABSTINENCIA

$$
\begin{array}{r}
(N=41) \\
46 \% \\
12 \% \\
20 \% \\
12 \% \\
10 \% \\
(N=35) \\
66 \% \\
34 \% \\
(N=28) \\
61 \% \\
39 \% \\
(N=35) \\
51 \% \\
49 \% \\
(N=29) \\
59 \% \\
41 \%
\end{array}
$$

\section{COMORBILIDAD}

$(N=120)$

$44 \%$

$7 \%$

$22 \%$

$10 \%$

$17 \% \quad$ chi $=2.57$

( $N=89$ )

$46 \%$

$54 \%$ chi $=3.87 *$

$(N=73)$

$44 \%$

$56 \% \quad$ chi $=2.30$

$(N=98)$

$39 \%$

$61 \%$ chi $=1.69$

$(N=83)$

$57 \%$

$43 \% \quad$ chi $=0.03$

${ }^{*} p<0.05$ 
(42), nos llevaría a pensar que no es la comorbilidad en sí, sino el tipo de trastorno asociado y su respuesta al tratamiento y evolución lo que puede influir en la respuesta al tratamiento y evolución de la dependencia alcohólica.

Nuestros resultados cuestionan que la comorbilidad, tomada en su conjunto, influya en la respuesta al tratamiento y en la evolución a largo plazo y que pueda servir para establecer subtipos de dentro de la dependencia alcohólica. Los resultados son más indicativos de que no existen características específicas de los dependientes del alcohol con comorbilidad asociada; sino que las diferencias sociodemográficas o clínicas que pueden aparecer están en función del tipo de trastorno asociado (51), que pueden matizar la presentación de la dependencia alcohólica, pero no la modifica de manera sustancial, que van a requerir más necesidades asistenciales, y que su abordaje terapéutico se debe basar en intervenciones específicas e independientes para cada uno de los trastornos, y que lo deseable es que estas intervenciones se lleven a cabo de manera integrada y por un único equipo terapéutico (52).

\section{BIBLIOGRAFIA}

1. O'Sullivan K, Whillans P, Daly M, Carroll B, Clare A, Cooney, J. A comparison of alcoholics with and without coexisting affective disorder. Br J Psychiatry, 1983; 143: 133-138.

2. Schuckit MA. The clinical implicatios of primary diagnostic groups among alcoholics. Arch Gen Psychiatry 1985; 42: 1043-1049.

3. Schuckit MA, Tipp JE, Bergman M, Reich W, Hesselbrock VM, Smith TL. Comparisson of induced and independent major depressive disorders in 2945 alcoholics, Am J Psychiatry 1997; 154: 948-957.

4. Lewis CE, Rice J, Helzer JE. Diagnostic interactions. Alcoholism and antisocial personality. J Nerv Ment Dis 1983; 171 (2): 105-111.

5. Liskow B, Powell BJ, Nickel E, Penick E. Antisocial alcoholics are there clinically significant diagnostic subtypes.

J Stud Alcohol 1991; 52: 62-69.

6. Kushner M, Sher K, Beitman B. The relation between alcohol problems and the anxiety disorders. Am J Psychiatry 1990; 146: 685-695.

7. Suzuki K, Higuchi S, Yamada K, Mizutani Y, Kono, H. Young female alcoholics with and without eating disorders: A comparative study in Japan. Am J Psychiatry 1993; 150: 1053-1058.

8. Regier DA, Farmer ME, Rae DS, Locke BZ, Keith SJ, Judd LL, Goodwin FK. Comorbidity of mental disorders with alcohol and other drug abuse. Results from the Epidemiologic Catchment Area (ECA) study. JAMA 1990; 264: 2511-2518.
9. Kessler RC, McGonagle KA, Zhao S, Nelson CB, Hughes M, Eshleman S, Wittchen HV, Kendler KS. Lifetime and 12-month prevalence of DSM III-R psychiatric disorders in the United States. Results from the National Comorbidity Survey. Arch Gen Psychiatry 1994; 51: 8-19.

10. Bland RC, Stephen CN, Russell JM, Orn HT (eds.). Epidemiology of psychiatric disorders in Edmonton: phenomenology and comorbidity. Acta Psychiatr Scand 1994; 89, suppl.376.

11. Ross HE. DSM III-R alcohol abuse and dependence and psychiatric comorbidity in Ontario: results from the mental health supplement to the Ontario Health Survey. Drug Alc Depend 1995; 39: 111-128.

12. Hesselbrock MN, Meyer RE, Keener JJ. Psychopathology in hospitalized alcoholics. Arch Gen Psychiatry 1985; 42: 1050-1055.

13. Penick EC, Powell BJ, Nickel EJ, Bingham SF, Riesenmy KR, Read MR, Campbell J. Comorbidity of lifetime psychiatric disorder among male alcoholic patients. Alc Clin Exp Res 1994; 18: 1289-1293.

14. Tomasson K, Vaglum P. A nationwide representative sample of treatment seeking alcoholics: a study of psychiatric comorbidity. Acta Psychiatr Scand 1995; 12: 378-385.

15. Cuadrado, P. Un estudio de la comorbilidad psiquiátrica en la dependencia alcohólica. Actas Luso-Esp Neurol Psiquiatr 1998; 26: 173-179.

16. Powell BJ, Penick EC, Othmer E, Bingham SF, Rice AS. Prevalence of additional psychiatric syndromes among male alcoholics. J Clin Psychiatry 1982; 43: 404-407.

17. Penick EC, Powell BJ, Othmer E, Bingham SF, Rice AS, Liese BS. Subtyping alcoholics by coexisting psychiatric syndromes: Course, family history, outcome. En Goodwin DW, Van Dusen KT, Mednick SA (eds) Longitudinal research in alcoholism. Boston: Kluwer Nijhoff Publishing, 1984: 167- 196.

18. Penick EC, Nickel EJ, Cantrell PF, Powell BJ, Read MR, Thomas MM. The Emerging concept of dual diagnosis: An overview and implications. J Chem Depend Treat 1990; 3: 1-54.

19. Penick EC, Powell BJ, Liskow BI, Jackos JO, Nickel EJ. The stability of coexisting psychiatric syndromes in alcoholic men after one year. J Stud Alcohol 1988; 49: 395- 405.

20. Brady KT, Grice DE, Dustan L, Randall C. Gender differences in substance use disorders. Am J Psychiatry 1993; 150: 1707-1711.

21. Lewis CE, Bucholz KK, Spitznagel E, Shoyka JJ. Effects of gender and comorbidity on problem drinking in a community sample. Alc Clin Exp Res 1996; 20: 466476.

22. Ross HE, Glaser FB, Germanson T. The prevalence of psychiatric disorders in patients with alcohol and other drug problems. Arch Gen Psychiatry 1988; 45: 10231031.

23. Hasin DS, Grant BF, Endicott J. Lifetime psychiatric comorbidity in hospitalized alcoholics: Subjects and 
familial correlates. Int J Addiction 1988; 23 (8): 827850.

24. Blow FC, Cook CAL, Booth BM, Falcon JP, Friedman MJ. Age- related psychiatric comorbidities and level of functioning in alcohol veterans seeking outpatient treatment, Hosp Comm Psychiatry 1992; 43: 990-999.

25. Kessler RC, Crum RM, Warner CA, Nelson CB, Schulenberg J, Anthony JC. Lifetime Co-occurrence of DSM III$\mathrm{R}$ alcohol abuse and dependence with other psychiatric disorders in the National Comorbidity Survey. Arch Gen Psychiatry 1997; 54: 313-321.

26. Westreich L, Guedj P, Galanter M, Baird D. Differences between men and women in dual-diagnosis treatment. Am J Addiction 1997; 6 (4): 311-317.

27. Powell BJ, Penick EC, Nickel EJ, Liskow BI, Riesenmy KD, Campion SL, Brown EF. Outcomes of co-morbid alcoholic men. A 1-year follow-up, Al Clin Exp Res 1992; 16: 131-138.

28. Kesch $\mathrm{KL}$, Klein DN. The relatioship between age at onset and comorbidity in psychiatric disorders. J Nerv Ment Dis 1996; 184: 703-707.

29. Cook BL, Winokur G, Fowler RC, Liskow BI. Classification of alcoholism with reference to comorbidity. Compr Psychiatry 1994; 35: 165-170.

30. McGue M, Shutsake W, Taylor J, lacono WG. Personality and substance use disorders. I. Effects of gender and alcoholism subtype. Alc Clin Exp Res 1997; 21 (3): 513-520.

31. Roy A, DeJong J, Lamparski D, Adinoff B, George T, Moore V, Garrett D, Kerich M, Linnoila M. Mental disorders among alcoholics. Relationship to age of onset and cerebrospinal fluid neuropeptides. Arch Gen Psychiatry $1991 ; 48:$ 423-427.

32. Rimmer J, Reich T, Winokur G. Alcoholism: V. Diagnosis and clinical variation among alcoholics. $\mathbf{Q}$ J Stud Alcohol 1972; 33: 658-666.

33. Lewis CE, Robins L, Rice J. Association of alcoholism with antisocial personality in urban men. J Nerv Ment Dis 1985; 173: 166-174.

34. Morgenstern J, Langenbucher J, Labouvie E, Miller KJ. The comorbidity af alcoholism and personality disorders in a clinical population: prevalence rates and relation to alcohol typology variables. J Abnorm Psychol 1997; 106: 74-84.

35. Kendler KS, Davis CG, Kessler RC. The familial aggregation of common psychiatric and substance use disorder in the National Comorbidity Survey: a family study. Br J Psychiatry 1997; 170: 541-548.

36. O'Sullivan K, Rynne C, Miller J, O'Sullivan S, Fitzpatrick V, Hux M, Cooney J, Clare A. A follow-up study on alcoholics with and without co-existing affective disorder. Br J Psychiatry 1988; 152: 813-819.
37. Ross H, Shirley M. Life-time problem drinking and psychiatric co-morbidity among Ontario women. Addiction 1997; 98: 183-196.

38. Dickey B, Azeni H. Persons with dual diagnosis of substance abuse and major mental illness: their excess costs of psychiatric care. Am J Public Health 1996; 86: 973-977.

39. Rounsaville BJ, Dolinsky ZS, Babor TF, Meyer RE. Psychopathology as a predictor of treatment outcome in alcoholics. Arch Gen Psychiatry 1987; 44: 505-513.

40. Verhene R, van den Brink W, Hartgers C. Personality disorders predict relapse in alcoholic patients, Addic Behav 1998; 23 (69): 809-822.

41. Kranzler HR, Del Boca FK, Rounsaville BJ. Comorbid psychiatric diagnosis predicts three-year outcomes in alcoholics. A posttreatment natural history study. J Stud Alcohol 1996; 57: 619-626.

42. Brown TG, Seraganian P, Tremblay J. Alcohol and cocaine abusers 6 months after traditional treatment: do they fare as well as problem drinkers ?. J Subs Abuse Treat 1993; 10: 545-552.

43. Loosen PT, Dew BW, Prange AJ. Long-term predictors of outcome in abstinent alcoholic men. Am J Psychiatry 1990; 147: 1662-1666.

44. Kranzler HR, Del Boca FK, Rounsaville BJ. Comorbid psychiatric diagnosis predicts three-year outcomes in alcoholics. A posttreatment natural history study, J Stud Alcohol 1996; 57: 619-626.

45. Sellman JD, Joyce PR. Does depression predict relapse in the 6 months following treatment for men with alcohol dependence. Austr N Zel J Psychiatry 1996; 30: 573-578.

46. Eysenck HJ, Eysenck SBG. Cuestionario de Personalidad para niños y adultos. Madrid: TEA Ediciones SA, 1986.

47. Miller WR, Marlatt GA. Manual for the Comprehensive Drinker Profile. Odessa, Florida: Psychological Assesment Resources Inc, 1984.

48. Cuadrado P, García R, Pata MD, Noval MJ. Respuesta de la dependencia alcohólica a un programa comunitario de tratamiento. Psiquiatría Pública 1998; 10 (3): 162-168.

49. SPSS INC. SPSS/PC + Update for V 3.1. Chicago: SPSS INC ed., 1984.

50. Cuadrado P. Evolución de la dependencia alcohólica en tratamiento. Factores predictivos en un seguimiento de 5 a 7 años. Adicciones 1998; 10: 335-341.

51. Cuadrado P. Enfermos con dependencia al alcohol y comorbilidad psiquiátrica: un grupo heterogéneo. Actas Esp Psiquiatr 1999; 27: 235-244.

52. Cuadrado P. Comorbilidad psiquiátrica en la dependencia alcohólica. II. Implicaciones clínicas y terapéuticas.

Actas Luso-Esp Neurol Psiquiatr 1997; 25: 34-44. 
